\title{
Phenytoin Induced DRESS Syndrome
}

\author{
Nayan Mani Deka • Rashna Dass • Bipul Kumar Das • \\ Rejaul Hoque
}

Received: 6 January 2012 / Accepted: 30 March 2012 / Published online: 29 April 2012

(C) Dr. K C Chaudhuri Foundation 2012

Sir,

Drug rash with eosinophilia and systemic symptoms (DRESS) is a severe form of drug reaction characterized by fever, skin rash, lymphadenopathy, hematological abnormalities, and internal organ involvement 2 to $8 \mathrm{wk}$ after a drug is first used [1]. A 10-y-old boy with epilepsy was admitted with high fever, cough, breathing difficulty, generalized rash and hoarseness of voice. He was on gabapentin, clobazam and phenytoin. He was febrile, tachypneic, had a generalized erythematous, scaly and itchy maculopapular rash, oral mucosal ulceration, facial and neck swelling, bilateral periorbital and extremity edema, generalized lymphadenopathy, non-tender hepatomegaly and chest rales. Tests showed eosinophilia, mild elevation of liver enzymes and bilateral paracardiac opacity. He was started on intravenous amoxyclavulanic acid initially and then on vancomycin following growth of Staphylococcus aureus in blood culture. Fever persisted even after $72 \mathrm{~h}$ of vancomycin. Bone marrow examination for suspected macrophage activation syndrome (MAS) was normal. Other targeted investigations for MAS were negative. In view of phenytoin therapy with the associated findings, he was diagnosed as DRESS syndrome. Phenytoin was stopped and systemic corticosteroid @ 2 mg/kg/d was started. There was dramatic

N. M. Deka $\cdot$ R. Dass $\cdot$ B. K. Das $\cdot$ R. Hoque

Department of Pediatric Disciplines, North Eastern Indira Gandhi Regional Institute of Health and Medical Sciences (NEIGRIHMS), Mawdiangdiang,

Shillong, Meghalaya, India

R. Dass $(\bowtie)$

Department of Pediatric Disciplines, North Eastern Indira Gandhi Regional Institute of Health and Medical Sciences (NEIGRIHMS), Room no. 218, PO: Mawdiangdiang,

Shillong 793018, India

e-mail: rashnadass@gmail.com improvement with defervescence of fever within $48 \mathrm{~h}$, improvement in general condition and disappearance of rashes. An adverse drug reaction complicates about $5 \%$ to $15 \%$ of patients [2]. The incidence of DRESS following anticonvulsant therapy varies from 1 in 1,000 to 1 in 100,000 exposures [3]. The mechanism is either secondary to circulating antibodies or toxic metabolites. There is no gold standard for diagnosis, and two diagnostic criteria, the RegiSCAR criteria [4] and the Japanese consensus group criteria [5] have been proposed. Differential diagnoses include Stevens-Johnson syndrome (SJS), toxic epidermal necrolysis (TEN) and pustular erythematous drug eruptions in general. These occur about 1 to $3 \mathrm{wk}$ after start of the offending drug without eosinophilia and atypical lymphocytosis. Withdrawal of the offending drug is the primary treatment. Systemic corticosteroids help in resolution of the symptoms, although the rash and hepatitis may persist for several weeks. Early recognition of DRESS syndrome and withholding and/or changing the medication is necessary to prevent potentially fatal outcomes.

\section{References}

1. Tas S, Simonart T. Management of drug rash with eosinophilia and systemic symptoms (DRESS syndrome): an update. Dermatology. 2003;206:353-6.

2. Jick H. Adverse drug reactions: the magnitude of the problem. J Allergy Clin Immunol. 1984;74:555-7.

3. Vittorio CC, Muglia JJ. Anticonvulsant hypersensitivity syndrome. Arch Intern Med. 1995;155:2285-90.

4. Kardaun SH, Sidoroff A, Valeyrie-Allanore L, et al. Variability in the clinical pattern of cutaneous side-effects of drugs with systemic symptoms: does a DRESS syndrome really exist? $\mathrm{Br} J$ Dermatol. 2007;156:609-11.

5. Shiohara T, Iijima M, Ikezawa Z, Hashimoto K. The diagnosis of DRESS syndrome has been sufficiently established on the basis of typical clinical features and viral reactivations. $\mathrm{Br} \mathrm{J}$ Dermatol. 2007;156:1083-4. 